\title{
Notificação de violência: conhecimento de cirurgiões-dentistas que atuam na Região Carbonífera, SC
}

\author{
Tamiris Bonfim Fernandes*; Mayara Pedroso Rocha*; Ana Regina da Silva Losso**; Fernanda \\ Guglielmi Faustini Sonego**** \\ * Discente de Graduação em Odontologia, Universidade do \\ Extremo Sul Catarinense, Criciúma, SC \\ ** Docente do curso de Enfermagem, Universidade do Extremo Sul \\ Catarinense, Criciúma, SC \\ *** Docente do curso de Odontologia, Universidade do Extremo Sul \\ Catarinense, Criciúma, SC
}

Recebido em 20/07/2017. Aprovado em 22/10/2017.

\begin{abstract}
RESUMO
O objetivo do estudo foi analisar o conhecimento e conscientizar cirurgiões-dentistas sobre a notificação compulsória de violência interpessoal/autoprovocada. Tratou-se de um estudo quantitativo, do tipo transversal e de análise descritiva, desenvolvido nos doze municípios da Região Carbonífera, localizada no sul catarinense, com os cirurgiões-dentistas da rede pública. Da população de 134 cirurgiões-dentistas, 108 (80,6\%) responderam ao questionário. Destes, 56 (52,8\%) eram do sexo masculino; 61 (56,5\%) estudaram em universidade pública; 27 (25\%) estão formados há mais de 21 anos e $21(19,6 \%)$ possuem especialização em Saúde Coletiva. Sobre o conhecimento dos profissionais acerca da ficha de notificação de violência, apenas 21,3\% $(n=23)$ relataram conhecê-la. Em relação aos tipos de violência, a física foi a mais citada pelos dentistas. No teste de Qui-quadrado de Pearson, observou-se significância estatística na associação do gênero feminino com possuir mais conhecimento sobre a ficha de notificação $(\mathrm{p}=0,036)$. Verificou-se que os profissionais desconhecem tanto a ficha de notificação quanto o banco de dados desse instrumento $(\mathrm{p}=<0,001)$, revelando, também, que a maioria nunca participou de atividade educativa sobre a temática $(\mathrm{p}=<0,001)$. Conclui-se que os cirurgiões-dentistas que atuam na rede pública dos municípios da região carbonífera demostraram pouco conhecimento quanto aos tipos de violência, a notificação compulsória de violência e ao encaminhamento das vítimas, porém, eles demonstraram interesse em aprender sobre o tema, pois participaram ativamente da atividade educativa realizada posteriormente à coleta de dados.
\end{abstract}

Descritores: Conhecimento. Violência. Sistemas de Informação em Saúde. Odontologia. 


\section{INTRODUÇÃO}

A violência pode ser definida como a ação desempenhada por indivíduos, grupos, classes ou nações, causando danos físicos, emocionais ou morais, a si ou a outros. É considerada violência, também, quando ocorre omissão, quando se nega ajuda, e/ou a negligencia ${ }^{1}$.

A Organização Mundial da Saúde (OMS) classifica três grupos, de acordo com quem comete $\mathrm{o}$ ato violento em violência contra si mesmo (autoprovocada ou autoinfligida); violência interpessoal (doméstica e comunitária); e violência coletiva (grupos políticos, organizações terroristas, milícias). Além disto, ela faz distinções sobre as naturezas da violência, denominando-as violência física; violência psicológica/moral; tortura; violência sexual; tráfico de seres humanos; violência financeira/ econômica; negligência/abandono; trabalho infantil; e intervenção legal ${ }^{2}$.

Em todo o mundo são registradas, anualmente, mais de 1,3 milhões de mortes em decorrência da violência, seja ela autoprovocada, interpessoal ou coletiva, correspondendo a $2,5 \%$ da mortalidade global ${ }^{3}$.

No Brasil, a violência é considerada um problema de saúde pública, que tem provocado forte impacto na morbimortalidade da população. No ano de 2001 foi publicada a portaria da Política Nacional de Redução de Morbidade por Acidentes e Violências, com objetivo de reduzir estes agravos. Em 2008 foi criada a ficha de notificação compulsória de violência e um instrutivo para o seu correto preenchimento, consolidando a implantação dessa política ${ }^{2}$. A notificação compulsória é determinada a todos os profissionais de saúde ou responsáveis por estabelecimentos públicos e privados de saúde, que prestam assistência ao paciente, conforme o artigo $8^{\circ}$ da Lei $n^{\circ} 6.259$, datada de 30 de outubro de $1975^{4}$.
Os profissionais de saúde têm dificuldade em realizar a notificação como conduta padrão, ainda que exista a obrigatoriedade legal, pois enfrentam como obstáculo o conhecimento dos tipos de violência e, consequentemente, seu reconhecimento. A notificação é um passo fundamental e uma estratégia de organização que propicia a construção de uma rede de atenção para o seu controle ${ }^{5,6}$.

É fundamental o conhecimento de todos os profissionais da saúde sobre os tipos de violência e a notificação de agravo, em especial os cirurgiões-dentistas, uma vez que a quantidade de lesões físicas na região de cabeça e pescoço é elevada. Quase metade das vítimas de abuso sexual pode apresentar lesões na cavidade oral, independente de terem lesões em seus órgãos genitais, salientando a importância de os cirurgiões-dentistas estarem atentos ao exame clínico de seus pacientes ${ }^{7}$.

Em estudos anteriores, com cirurgiõesdentistas que atuam na saúde pública, verificouse que, ao analisar a percepção e atitude dos mesmos frente à violência intrafamiliar, 74\% relataram não saber notificá-la e 70\% desconheciam o assunto. Apenas 26\% dos cirurgiões-dentistas notificaram casos de violência por eles diagnosticados, enfatizando que grande parte dos participantes desconhece este tema ${ }^{8}$. Assim, questiona-se se o mesmo não ocorre com os cirurgiões-dentistas de outros municípios. Será que eles notificam as suspeitas e/ou as confirmações de violência? Caso notifiquem, será que realizam os procedimentos corretamente? O presente estudo objetivou analisar o conhecimento de cirurgiões-dentistas que atuam nos municípios pertencentes à Associação dos Municípios da Região Carbonífera, no sul de Santa Catarina, sobre a notificação compulsória de violência interpessoal/autoprovocada. 


\section{METODOLOGIA}

Tratou-se de um estudo transversal e descritivo, originado a partir de um projeto de extensão, e que obteve aprovação do Comitê de Ética em Pesquisa com Seres Humanos, parecer $\mathrm{n}^{\mathrm{o}} 1.795 .249 / 2016$.

O estudo foi realizado nos doze municípios pertencentes à Associação dos Municípios da Região Carbonífera (AMREC), no sul de Santa Catarina, no período de julho de 2016 a junho de 2017.

A Região Carbonífera é composta pelos seguintes municípios: Balneário Rincão, Cocal do Sul, Criciúma, Forquilhinha, Içara, Lauro Müller, Morro da Fumaça, Nova Veneza, Orleans, Siderópolis, Treviso e Urussanga, totalizando 425.603 habitantes em toda a região9 .

A população em estudo foi composta por 134 cirurgiões-dentistas da rede pública, que possuíam registro no Cadastro Nacional de Estabelecimentos de Saúde (CNES), independente do vínculo empregatício. Após a assinatura do Termo de Consentimento Livre e Esclarecido, conforme preconiza a resolução $n^{\circ}$ 466/12 do Conselho Nacional de Saúde. Foram excluídos aqueles que estiveram ausentes por qualquer motivo no período da coleta de dados.

Foi utilizado um questionário semiestruturado, anônimo e autoaplicado, baseado no estudo de Luna et al. ${ }^{10}$. O instrumento continha trinta e duas questões fechadas e uma questão aberta para análise do conhecimento e, também, para nortear a atividade educativa realizada posteriormente.

Os dados foram armazenados em planilhas do software Microsoft Excel 2012 e são apresentados na forma de estatísticas descritivas e tabelas. Por meio do software Statistical Package for the Social Sciences (SPSS) versão 23, foi realizada a análise estatística, considerando nível de significância $\alpha=0,05$ e intervalo de confiança de $95 \%$.
O teste de Qui-quadrado de Pearson foi utilizado para quantificar a associação ou independência entre o conhecimento sobre a ficha de notificação, perfil do profissional, participação em alguma atividade educativa, se já se deparou com algum caso de violência, se realizou a notificação do caso, e as demais variáveis independentes. Para todos os testes supracitados foi

Posteriormente foi efetuada uma atividade educativa, como forma de padronizar medidas, fortalecer ações e qualificar o serviço, empregando metodologias ativas acerca do tema. Um folder educativo foi confeccionado, entregue ao final como reforço do conteúdo trabalhado, contendo os contatos específicos das instituições que auxiliam a rede e que são referências em cada município, para encaminhamentos das notificações compulsórias de violência.

\section{RESULTADOS}

Dos 134 cirurgiões-dentistas que atuam na rede pública nos doze municípios da AMREC, 80,6\% ( $\mathrm{n}=108)$ responderam o questionário, porém nem todas as questões foram respondidas pelos entrevistados.

Ao analisar o perfil dos participantes, observou-se maior número de homens $(52,8 \%$; $\mathrm{n}=56$ ), com renda familiar entre 5.001 e 10.000 reais $(41,3 \% ; n=43)$, na faixa etária entre 31 e 40 $\operatorname{anos}(37 \% ; n=40)$, casados $(63 \% ; n=68)$ e com filhos 51,9\% $(n=56)$. Pouco mais da metade dos cirurgiões-dentistas estudou em universidade pública $(56,5 \% ; n=61)$, sendo que $25 \%(n=27)$ deles estão formados há mais de 21 anos e 19,6\% $(\mathrm{n}=21)$ possuem especialização em Saúde Coletiva. Em relação ao tempo de serviço, 36,4\% $(\mathrm{n}=39)$ atuam há 2 anos no município. A maioria possui outro trabalho na área da saúde $(66,4 \%$; $\mathrm{n}=71)$, sendo, em grande parte, no setor privado $(88 \% ; \mathrm{n}=66)$.

Sobre o conhecimento dos cirurgiões- 
dentistas referente aos tipos de violência, a física foi a mais citada, sendo considerada como violência por $100 \%(\mathrm{n}=107)$ dos respondentes. Apenas $21,3 \%(n=23)$ relataram conhecer a ficha de notificação compulsória de violência e somente 9,4\% ( $n=10)$ conhecem algum banco de dados sobre a mesma. Sobre ter presenciado algum tipo de violência, 60,4\% (n=64) já presenciaram, 40,2\% (n=43) já se depararam com algum caso de violência durante a sua atuação profissional, e somente $15 \% \quad(n=16)$ relataram conhecer a rede de assistência às vítimas de violência. Em relação ao medo de envolvimento legal, ao realizar uma notificação de violência, $35 \%(n=36)$ dos entrevistados afirmaram possuir, motivo pelo qual apenas $11,8 \%(n=6)$ notificaram o caso para algum órgão da rede (tabela 1).

Tabela 1. Conhecimento dos cirurgiões-dentistas sobre violência, nos municípios da AMREC, de julho de 2016 a junho de 2017

\begin{tabular}{lr}
\hline Variável (n) & $\mathbf{n}(\mathbf{\%})$ \\
\hline Considera a violência física uma violência $(\mathrm{n}=107)$ & $107(100,0)$ \\
Considera a violência psicológica/moral uma violência $(\mathrm{n}=107)$ & $103(96,3)$ \\
Considera a tortura uma violência $(\mathrm{n}=107)$ & $105(98,1)$ \\
Considera a violência sexual uma violência $(\mathrm{n}=107)$ & $105(98,1)$ \\
Considera o tráfico de seres humanos uma violência $(\mathrm{n}=107)$ & $99(92,5)$ \\
Considera a violência financeira/econômica uma violência $(\mathrm{n}=107)$ & $86(80,4)$ \\
Considera a negligência/abandono uma violência $(\mathrm{n}=107)$ & $99(92,5)$ \\
Considera o trabalho infantil uma violência $(\mathrm{n}=107)$ & $97(90,7)$ \\
Considera a intervenção legal uma violência $(\mathrm{n}=107)$ & $80(74,8)$ \\
Já presenciou algum tipo de violência $(\mathrm{n}=106)$ & $64(60,4)$ \\
Costuma ler sobre a temática de violência $(\mathrm{n}=108)$ & $42(38,9)$ \\
Conhece a ficha de notificação de violência $(\mathrm{n}=108)$ & $23(21,3)$ \\
Como teve acesso a ficha de notificação de violência (n=23) & \\
$\quad$ Na graduação & $2(8,7)$ \\
$\quad$ Na pós-graduação & $2(8,7)$ \\
$\quad$ Pesquisa própria & $4(17,4)$ \\
Outros & $15(65,2)$ \\
Conhece algum banco de dados de notificação de violência $(\mathrm{n}=106)$ & $10(9,4)$ \\
A violência é uma temática discutida no trabalho $(\mathrm{n}=106)$ & $37(34,9)$ \\
Na sua atuação profissional já se deparou com caso de violência $(\mathrm{n}=107)$ & $43(40,2)$ \\
Notificou o caso (n= 51) & $6(11,8)$ \\
Tem medo de envolvimento legal ao notificar uma violência $(\mathrm{n}=103)$ & $36(35,0)$ \\
Ao notificar saberia onde encaminhar (n=106) & $36(35,0)$ \\
Conhece a rede de assistência às vítimas de violência (n=107) & $16(15,0)$ \\
\hline
\end{tabular}

*Considerou-se como $100 \%$ o número total de respondentes de cada questão

A tabela 2 apresenta a correlação entre conhecimento sobre a ficha de notificação de violência e demais variáveis. Observou-se significância estatística entre o conhecimento e o gênero dos profissionais, mostrando que o feminino era o que mais possuía conhecimento sobre a ficha de notificação $(\mathrm{p}=0,036)$. Quando relacionado com possuir especialização em
Saúde Coletiva, a significância estatística também foi observada, revelando que os dentistas que possuem esta especialização conhecem a ficha de notificação de violência $(\mathrm{p}=0,008)$, no entanto, a maioria dos profissionais que possuem especialização em outra área a desconhece $(\mathrm{p}=0,003) . \mathrm{Na}$ associação com os profissionais conhecerem 
algum banco de dados sobre a notificação, de notificação de violência quanto o banco de verificou-se que eles desconhecem tanto a ficha dados desse instrumento $(p=<0,001)$.

Tabela 2. Associação entre conhecimento sobre a ficha de notificação de violência e demais variáveis, nos municípios da AMREC, de julho de 2016 a junho de 2017

\begin{tabular}{|c|c|c|c|}
\hline \multirow{2}{*}{ Variável (n) } & \multicolumn{2}{|c|}{ Conhecimento $\mathrm{n} *(\%)$} & \multirow[t]{2}{*}{$\mathbf{p}$} \\
\hline & Sim & Não & \\
\hline \multicolumn{4}{|l|}{ Gênero $(n=106)$} \\
\hline Masculino & $6(27,3)$ & $44(52,4)$ & 0,036 \\
\hline Feminino & $16(72,7)$ & $40(47,6)$ & \\
\hline \multicolumn{4}{|c|}{ Possui especialização em Saúde Coletiva $(n=107)$} \\
\hline Sim & $9(39,1)$ & $12(14,3)$ & 0,008 \\
\hline Não & $14(60,9)$ & $72(85,7)$ & \\
\hline \multicolumn{4}{|c|}{ Possui especialização em outra área $(n=106)$} \\
\hline Sim & $8(34,8)$ & $58(69,0)$ & 0,003 \\
\hline Não & $15(65,2)$ & $26(31,0)$ & \\
\hline \multicolumn{4}{|c|}{ Possui residência em Saúde Coletiva $(n=107)$} \\
\hline Sim & $2(8,7)$ & $1(1,2)$ & 0,053 \\
\hline Não & $21(91,3)$ & $83(98,8)$ & \\
\hline \multicolumn{4}{|c|}{ Possui outro trabalho na área da saúde $(n=107)$} \\
\hline Sim & $13(56,5)$ & $58(69,0)$ & 0,043 \\
\hline Não & $8(34,8)$ & $25(29,8)$ & \\
\hline Sim, em outro setor & $2(8,7)$ & $0(0,0)$ & \\
\hline \multicolumn{4}{|c|}{ Conhece algum banco de dados de notificação $(n=106)$} \\
\hline $\operatorname{Sim}$ & $9(40,9)$ & $1(1,2)$ & $<0,001$ \\
\hline Não & $13(59,1)$ & $83(98,8)$ & \\
\hline \multicolumn{4}{|c|}{ A violência é uma temática discutida no trabalho $(n=106)$} \\
\hline Sim & $14(60,9)$ & $23(27,7)$ & 0,003 \\
\hline Não & $9(39,1)$ & $60(72,3)$ & \\
\hline \multicolumn{4}{|c|}{ Identificou a violência com o relato da vítima $(n=44)$} \\
\hline Sim & $2(16,7)$ & $16(50,0)$ & 0,045 \\
\hline Não & $10(83,3)$ & $16(50,0)$ & \\
\hline \multicolumn{4}{|l|}{ Notificou o caso $(n=51)$} \\
\hline Sim & $3(21,4)$ & $3(8,1)$ & 0,188 \\
\hline Não & $11(78,6)$ & $34(91,9)$ & \\
\hline \multicolumn{4}{|c|}{ Ao notificar saberia para onde encaminhar $(n=106)$} \\
\hline Sim & $19(82,6)$ & $11(13,3)$ & $<0,001$ \\
\hline Não & $4(17,4)$ & $72(86,7)$ & \\
\hline \multicolumn{4}{|c|}{ Conhece a rede de assistência à violência $(n=107)$} \\
\hline Sim & $13(56,5)$ & $3(3,6)$ & $<0,001$ \\
\hline Não & $10(43,5)$ & $81(96,4)$ & \\
\hline
\end{tabular}

*Considerou-se como $100 \%$ o número total de respondentes de cada questão.

De acordo com os dados sobre os profissionais que já se depararam com algum caso de violência durante a sua atuação profissional, constatou-se que a maioria dos dentistas já presenciou alguma violência durante a sua atuação $(\mathrm{p}=<0,001)$. Houve também uma significância estatística entre o profissional que já presenciou violência em casa $(\mathrm{p}=0,043)$. Ainda 
que a relação não tenha sido estatisticamente significante com os profissionais que identificaram o caso pelo comportamento alterado da vítima, o estudo mostrou que a maioria dos cirurgiões-dentistas que já se deparou com violência não a identificou por meio do comportamento alterado da vítima $(\mathrm{p}=0,422)$, porém, existiu uma associação significativa com a identificação de violência por outros meios $(\mathrm{p}=0,046)$. Contudo, quando questionados se possuíam medo de envolvimento legal ao realizar uma ficha de notificação de violência, houve uma associação significativa, sugerindo que os profissionais que já se deparam, relatam não possuir medo de envolvimento legal $(\mathrm{p}=0,043)$ (tabela 3).

Tabela 3. Associação entre ter se deparado com casos de violência na atuação profissional e outras variáveis, nos municípios da AMREC, de julho de 2016 a junho de 2017

\begin{tabular}{|c|c|c|c|}
\hline \multirow{2}{*}{ Variável (n) } & \multicolumn{2}{|c|}{ Se deparou $n *(\%)$} & \multirow{2}{*}{$\mathbf{p}$} \\
\hline & Sim & Não & \\
\hline \multicolumn{4}{|c|}{ Presenciou algum tipo de violência $(n=105)$} \\
\hline Sim & $37(86,0)$ & $26(41,9)$ & $<0,001$ \\
\hline Não & $6(14,0)$ & $36(58,1)$ & \\
\hline \multicolumn{4}{|c|}{ Presenciou a violência em casa $(n=65)$} \\
\hline Sim & $5(13,5)$ & $0(0,0)$ & 0,043 \\
\hline Não & $32(86,5)$ & $28(100,0)$ & \\
\hline \multicolumn{4}{|c|}{ Presenciou a violência no trabalho $(n=65)$} \\
\hline Sim & $21(56,8)$ & $4(14,3)$ & $<0,001$ \\
\hline Não & $16(43,2)$ & $24(85,7)$ & \\
\hline \multicolumn{4}{|c|}{ Identificou o caso pelo comportamento alterado da vítima $(n=44)$} \\
\hline Sim & $17(39,5)$ & $0(0,0)$ & 0,422 \\
\hline Não & $26(60,5)$ & $1(100,0)$ & \\
\hline \multicolumn{4}{|c|}{ Identificou a violência de outra maneira $(n=44)$} \\
\hline Sim & $8(18,6)$ & $1(100,0)$ & 0,046 \\
\hline Não & $35(81,4)$ & $0(0,0)$ & \\
\hline \multicolumn{4}{|c|}{ Medo de envolvimento legal $(n=102)$} \\
\hline Sim & $20(46,5)$ & $16(27,1)$ & 0,043 \\
\hline Não & $23(53,5)$ & $43(72,9)$ & \\
\hline
\end{tabular}

*Considerou-se como $100 \%$ o número total de respondentes de cada questão.

Conforme os dados apresentados sobre a notificação dos casos de violência realizada pelos profissionais, houve associação significativa com o tempo de trabalho do cirurgião-dentista no município, salientando que os profissionais que mais notificam são os que trabalham há menos tempo no município $(\mathrm{p}=0,029)$. Observou-se também que foi limítrofe a correlação estatística sobre presenciar violência no trabalho, demonstrando que a maioria dos profissionais que presenciou a violência no trabalho não notificou o caso $(\mathrm{p}=0,056)$ (tabela 4$)$.
A respeito da participação dos profissionais em atividades educativas sobre $\mathrm{o}$ tema, houve relevância significativa quando associado ao tempo de formado, mostrando que os profissionais que mais participaram possuíam entre 3 e 10 anos de formados $(p=0,016)$. Houve também significância quando a participação na atividade educativa foi correlacionada aos profissionais que possuíam residência em saúde coletiva, indicando que os que tinham residência participaram mais das atividades educativas $(\mathrm{p}=0,044)$. 
Apesar de não ter expressado relevância estatística quando esta participação na atividade foi associada ao tempo de trabalho na saúde pública, os dados demonstraram que, quanto mais tempo de trabalho, menor a participação em atividades educativas $(\mathrm{p}=0,639)$. Verificou-se, ainda, significância com o conhecimento da ficha de notificação de violência, revelando que a maioria que desconhece a ficha de violência nunca participou de atividade educativa sobre a temática $(\mathrm{p}=<0,001)$. Em contrapartida, os que mais conhecem a rede são os dentistas que participaram de atividades educativas sobre $o$ tema $(\mathrm{p}=<0,001)$.

Tabela 4. Associação entre notificar o caso identificado e outras variáveis, nos municípios da AMREC, de julho de 2016 a junho de 2017

\begin{tabular}{lccc}
\hline \multicolumn{1}{c}{ Variável (n) } & \multicolumn{2}{c}{ Notificou $\mathbf{n}^{*(\%)}$} & \multirow{2}{*}{ Pim } \\
\hline Tempo de trabalho como CD no município $(n=51)$ & & Não & \\
0 a 2 anos & $4(66,7)$ & $13(28,9)$ & 0,029 \\
3 a 5 anos & $0(0,0)$ & $5(11,1)$ & \\
6 a 8 anos & $0(0,0)$ & $8(17,8)$ & \\
9 a 11 anos & $0(0,0)$ & $4(8,9)$ & \\
12 a 14 anos & $1(16,7)$ & $0(0,0)$ & \\
> 15 anos & $1(16,7)$ & $15(33,3)$ & \\
Presenciou violência no trabalho $(n=41)$ & $5(100,0)$ & $20(55,6)$ & 0,056 \\
Sim & $0(0,0)$ & $16(44,4)$ & \\
Não & & & \\
Busca ler sobre o tema em Teses $(n=24)$ & $1(33,3)$ & $0(0,0)$ & 0,007 \\
Sim & $2(66,7)$ & $21(100,0)$ & \\
Não & & & \\
*Considerou-se como 100\% o número total de respondentes de cada questão. & &
\end{tabular}

Após a coleta de dados foi realizada uma atividade educativa em cada município, baseada nas dificuldades encontradas. Esta atividade foi avaliada pelos participantes, por meio de um questionário, expressando o nível de satisfação dos mesmos em participarem do estudo. As respostas foram predominantemente positivas.

\section{DISCUSSÃO}

A violência é atualmente reconhecida como um problema de saúde pública mundial. Este estudo almejou abordar o conhecimento do cirurgião-dentista que atua na rede pública dos municípios da região carbonífera catarinense. Os dados obtidos foram utilizados para realizar uma atividade educativa visando a qualificação dos serviços de saúde e dos profissionais, o atendimento adequado e humanizado, além de possibilitar medidas de prevenção.

Os resultados, do perfil do cirurgiãodentista, no nosso estudo mostraram que o tempo de formado é, em sua maioria, superior a 21 anos, porém, a ficha de notificação de violência foi criada em 2008, ou seja, muito tempo após a conclusão da graduação dos entrevistados, corroborando com outros estudos, e enfatizando o motivo pelo qual houve desconhecimento dos profissionais acerca do tema ${ }^{11,12}$.

A incapacidade do profissional em identificar uma violência pode ser aplicada a fatores como ausência ou carência de abordagem do tema na graduação e em treinamentos dos profissionais ou mesmo uma conduta clínica baseada no modelo biomédico ${ }^{13}$. Apesar de 
19,6\% (n=21) dos participantes possuírem especialização em Saúde Coletiva, a maior parte deles não tinha informação sobre a ficha de notificação compulsória. Portanto, é essencial que as Instituições de Ensino Superior deem maior ênfase ao combate e à prevenção da violência durante a formação dos profissionais, permitindo, assim, a melhora da assistência oferecida ao paciente e aumentando o incentivo à cultura de paz ${ }^{14}$.

Verificou-se no presente estudo que poucos profissionais relataram conhecer a rede de assistência às vítimas de violência. Porém, conhecer os diferentes serviços que compõem a rede de atenção e prevenção da violência é um passo de extrema importância ${ }^{15}$, pois não saber para onde encaminhar os casos de violência, acaba contribuindo para o quadro de subnotificação ${ }^{16}$.

A violência física é muitas vezes difícil de ser camuflada, sendo mais fácil identificá-la e notificá-la aos órgãos competentes ${ }^{17}$. Justifica-se pelo fato de que, neste estudo, a violência física foi a forma de violência mais considerada pelos profissionais. No entanto, um dos motivos associados à ausência de notificação de um caso de violência é justamente a dificuldade de identificação do agravo por parte dos profissionais de saúde ${ }^{18}$.

$\mathrm{Na}$ Odontologia é possível identificar a violência de várias formas: na realização de anamnese cuidadosa pode-se conferir se a história descrita da lesão está condizente com o ferimento que o paciente apresenta. Quando houver a existência de lacerações na cavidade oral, a presença de dentes fraturados, com alteração de cor ou com muita necessidade curativa, além de lesões de Doenças Sexualmente Transmissíveis (DST), petéquias e eritema em palato mole e duro, indicando sexo oral forçado, o cirurgião-dentista poderá suspeitar de violência $^{19}$. Através de exame clínico bem detalhado, o profissional consegue detectar alguns tipos de violência, e isso justifica o fato de não ter ocorrido uma associação estatisticamente significante em nosso estudo, com o fato de o cirurgião-dentista ter se deparado com violência durante a sua atuação, e a identificação da mesma pelo comportamento alterado da vítima, mas sim de outras maneiras.

Diante de um caso suspeito ou confirmado de violência, a ficha de notificação compulsória deve ser preenchida em duas vias, ficando uma cópia na unidade notificadora, juntamente com o prontuário do paciente, pois é um documento odontolegal, e a outra precisa ser encaminhada ao setor municipal responsável pela Vigilância Epidemiológica $^{2,20,21}$. No entanto, alguns motivos contribuem para a omissão dos profissionais da área da saúde diante dos casos de violência, tais como medo e ausência de conhecimento dos indícios e da obrigatoriedade da notificação ${ }^{7}$. Nesta pesquisa, dentre os cirurgiões-dentistas que disseram ter se deparado com a violência durante sua atuação profissional, poucos relataram ter medo de envolvimento legal ao realizar notificação de violência, demonstrando que a não realização das mesmas está associada à falta de conhecimento acerca do instrumento e do papel do profissional diante dos casos de violência.

Os dados demonstraram que a maioria dos profissionais que presenciou a violência no trabalho não notificou o caso, vindo ao encontro dos resultados verificados em outros estudos ${ }^{8,22}$, reafirmando o despreparo dos profissionais frente ao tema, pois a realização da notificação de violência é uma obrigatoriedade aos profissionais da saúde. Sua ausência caracteriza contravenção penal do profissional que for omisso e não comunicar o crime que tenha tido conhecimento por meio de seu trabalho ${ }^{23}$.

Demonstrou-se que os profissionais não tinham conhecimento satisfatório sobre os tipos 
de violência, sobre a notificação de violência e o encaminhamento das vítimas. Esse despreparo pode propiciar a reincidência da violência e o agravamento dos casos. Por isso, ressalta-se a importância da realização da capacitação sobre o tema, aperfeiçoando e desenvolvendo a aptidão e a sensibilidade para abordar, reconhecer $\mathrm{e}$ notificar, de forma adequada, os casos de violência ${ }^{6}$.

Observou-se que os profissionais que mais participaram de alguma atividade educativa referente à notificação compulsória de violência foram os graduados há menos tempo, ou seja, entre 3 e 10 anos. Entende-se, com isso, que eles são os que mais reconhecem a necessidade de procura pela qualificação e capacitação profissional. Por esta razão, o tema violência deve ser estimulado e inserido nos currículos acadêmicos, para que na graduação os futuros profissionais tenham proximidade com este problema $^{24}$.

Quanto à percepção sobre violência contra pessoas, os profissionais da área da saúde apresentaram resultados estatisticamente positivos, quanto ao conhecimento, após os cursos sobre a temática da violência, sentindo-se mais preparados para identificar e atender vítimas de violência ${ }^{25}$. Ocorreu um aumento no número de casos notificados em três anos e este dado foi atribuído à capacitação realizada com os profissionais de saúde ${ }^{26}$, ressaltando a necessidade de capacitações com os profissionais, como a realizada no nosso trabalho.

Ainda existem muitos desafios na solidificação da notificação compulsória enquanto ação cotidiana nos diversos serviços de saúde $^{27}$, porém, a notificação de violência ainda é um instrumento que precisa ser mais explorado e aproveitado no desenvolvimento de políticas sociais, programas e projetos, mais atuantes na prevenção e controle do fenômeno da violência ${ }^{28}$.
Para que isso ocorra, é necessário que os profissionais realizem a notificação de todos os casos suspeitos e/ou confirmados de violência. Sugere-se a captação de fomentos para novas pesquisas a fim de ampliar o número de cirurgiões-dentistas capacitados.

\section{CONCLUSÃO}

Observou-se pouco conhecimento dos cirurgiões-dentistas que atuam na rede pública dos municípios da região carbonífera quanto aos tipos de violência, a notificação compulsória de violência e ao encaminhamento das vítimas, porém, eles demonstraram interesse em aprender sobre o tema, pois participaram ativamente da atividade educativa realizada posteriormente à coleta de dados.

Enfatiza-se a importância de capacitar e conscientizar estes profissionais acerca da temática e da realização da notificação compulsória de violência, visando, assim, a promoção de saúde e a prevenção do agravo notificável de violências.

\section{ABSTRACT \\ Notification of violence: knowledge of dentists working in the coal-mining region, $S C$}

The objective of the study was to analyze the knowledge and to make dental surgeons aware of the compulsory notification of interpersonal/selfinflicted violence. This was a quantitative, crosssectional and descriptive analysis study carried out in the twelve municipalities in the coalmining region located in the south of Santa Catarina, with public health dentists. Out of 134 dentists, $108 \quad(80.6 \%)$ answered the questionnaire. Among these, 56 (52.8\%) were male; $61(56.5 \%)$ studied in a public university; $27(25 \%)$ have been graduated for more than 21 years and $21(19.6 \%)$ have attended postgraduation course in Public Health. Regarding the professionals' knowledge about the violence notification form, only $21.3 \%(\mathrm{n}=23)$ reported knowing it. Concerning the types of violence, 
physical violence was mentioned the most by dentists. In the Pearson's Chi-squared Test, statistical significance was observed associating the female gender with more knowledge about the notification form $(p=0.036)$. Results showed that the professionals are not aware of either the notification form or the database of this instrument ( $\mathrm{p}=<0.001)$, also showing that the majority of them have never participated in any educational activities on the subject $(\mathrm{p}=0.001)$. In conclusion, dental surgeons who work in the public health network in municipalities in the coal-mining region have shown little knowledge about the types of violence, compulsory notification of violence and referral of victims, but they have shown interest in learning about it, since they participated actively in the educational activity carried out after the data collection.

Descriptors: Knowledge. Violence. Health Information Systems. Dentistry.

\section{REFERÊNCIAS}

1. Dominguez B, Machado K. Lei Maria da Penha: limites ou possibilidades. Radis: comunicação em saúde. 2010 abr;92-11.

2. Brasil. Ministério da Saúde, Secretaria de Vigilância em Saúde. Instrutivo para preenchimento da ficha de notificação de violência interpessoal/autoprovocada. Brasília: Ministério da Saúde, 2014.

3. Organização Mundial de Saúde (OMS). Relatório mundial sobre a prevenção da violência; 2014.

4. Brasil. Ministério da Saúde. Portaria 204, de 17 de fevereiro de 2016. Define a Lista Nacional de Notificação Compulsória de doenças, agravos e eventos de saúde pública nos serviços de saúde públicos e privados em todo o território nacional, nos termos do anexo, e dá outras providências. Brasília: Ministério da Saúde, 2016.

5. Figueiredo MC, de Oliveira Cesar M, da Silva JP, Borba EM. Prevalência de mulheres vítimas de violência no município de Porto Alegre e a influência de suas variáveis no âmbito odontológico. RFO UPF. 2012 set./dez;17(3):254-60.

6. Garbin CAS, Rovida TAS, Costa AA, Garbin AJI. Reconhecimento e notificação de violência pelos profissionais da estratégia de saúde da família. Arch Health Invest. 2016;5(1):8-12.

7. Alves MA, da Fonseca BA, Soares TRC, de França AKA, Azevedo RN, Tinoco RLR. Importância do cirurgião-dentista no diagnóstico de abuso sexual infantil-revisão de literatura. RBOL. 2016;3(2):92-9.

8. Garbin CAS, Rovida TAS, Costa AA, Garbin AJI. Percepção e atitude do cirurgião-dentista servidor público frente à violência intrafamiliar em 24 municípios do interior do estado São Paulo, 2013-2014. Epidemiol Serv Saúde. 2016 Mar;25(1):179-86.

9. Instituto Brasileiro de Geografia e Estatística [Internet]. Cidades: Santa Catarina, 2016 [Acesso em 25 maio 2017]. Disponível em: http://www.cidades.ibge.gov.br/xtras/perfil. php?lang $=\& \operatorname{codmun}=422000 \&$ search $=$ sant a-catarina.

10. Luna GLM, Ferreira RC, Vieira LJES. Notificação de maus-tratos anças e adolescentes por profissionais da equipe saúde da família [dissertação]. Fortaleza: Universidade de Fortaleza; 2007.

11. Campos PCM. Odontopediatras e violência contra crianças e adolescentes: como eles atuam? Int J Sci Dent. 2010;16(34):49-54.

12. Silva KBG, Cavalcanti AFC, Cavalcanti AL. Maus-tratos infantis: conhecimentos e condutas dos cirurgiões-dentistas da Estratégia Saúde da Família de GuarabiraPB. REFACS 2017;5(suppl 1):108-17.

13. Moreira GAR, Rolim ACA, Saintrain MVL, Vieira LJES. Atuação do cirurgião-dentista na identificação de maus-tratos contra crianças e adolescentes na atenção primária. 
Saúde Debate. 2015;39:257-67.

14. Galindo NAL, Gonçalves CFG, Neto NMG, Santos SC, Santana CSC, Alexandre AC. Violência infanto-juvenil sob a ótica da enfermagem. Rev Enferm UFPE. 2017;11(3):1420-9.

15. Porto RTS, Bispo JP Jr, Lima EC. Violência doméstica e sexual no âmbito da Estratégia de Saúde da Família: atuação profissional e barreiras para o enfrentamento. Physis. 2014;24(3): 787-807.

16. Rolim ACA, Moreira GAR, Corrêa CRS, Vieira LJES. Subnotificação de maus-tratos em crianças e adolescentes na Atenção Básica e análise de fatores associados. Saúde debate. 2014;794-804.

17. Sousa GM, Damasceno KCF, Borges LCF. Estratificação dos tipos de violência notificados pelo SINAN, no município de Porto Nacional, TO, em 2014. Interface (Botucatu). 2016;(11):34-45

18. Cordeiro KCC, Santos RM, Gomes NP, Melo DS, Mota RS, Couto TM. Formação profissional e notificação da violência contra a mulher. Revista Baiana de Enfermagem. 2015;29(3):209-17.

19. Losso EM, Dalledone M, Duda JG, Bertoli FMP, Pizzatto E, Correr GM, Souza JF. Maus-tratos infantis: o papel dos cirurgiõesdentistas na proteção das crianças e adolescentes. Curitiba: Universidade Positivo; 2015.

20. Velasco LEL, Rosa GC, Baldasso RP, Fernandes MM, Oliveira RN, Oliveira AMG. Responsabilidade dos cirurgiões-dentistas frente a maus-tratos contra a criança - revisão de literatura. BJFS. 2015; 4(3): 294-309.

21. Souza CE, Rodrigues IFMM, Zocratto KBF, Oliveira CAS. Violência infantil e a responsabilidade profissional do cirurgiãodentista - revisão de literatura. RBOL. 2017; 4(1): 53-63.
22. Garbin CAS, Melo LMLL, Moimaz SAS, Garbin AJL, Rovida TAS. Violência intrafamiliar na rotina do agente comunitário de saúde. J Health Sci Inst. 2014;32(4):385-9.

23. Fernandes MM, Lima LNC. Notificação Compulsória. In: Daruge E, Daruge E Jr, Francesquini L Jr. Tratado de Odontologia Legal e Deontologia. 1 ed. Rio de Janeiro: Guanabara Koogan, 2016, p. 101-110.

24. Quadros MN, Kirchner RM, Hildebrandt LM, Leite MT, Costa MC, Sarzi DM. Situação da violência contra crianças e adolescentes no Brasil. Enfermería Global. 2016;(44):174-85.

25. Pereira S, Vianna LAC. Cursos de capacitação em prevenção da violência: o impacto sobre os profissionais do setor da saúde. Rev Esc Enferm USP. 2014; 48(2):315-20.

26. Veloso MM, Magalhães CM, Dell'Aglio DD, Cabral IR, Gomes MM. Notificação da violência como estratégia de vigilância em saúde: perfil de uma metrópole do Brasil. Ciênc Saúde Coletiva. 2013;18(5):1263-1272.

27. Cezar PK, Arpini DM. Notificação compulsória da violência como possível fator de proteção à infância e a adolescência. Adolesc Saúde. 2016;13(suppl 2):114-23.

28. Ferraz LF. A violência contra a criança e o adolescente: a importância da notificação compulsória como mecanismo de proteção social [trabalho de conclusão de curso]. Porto Alegre: Universidade Federal do Rio Grande do Sul. Curso de Serviço Social, Departamento de Psicologia; 2016.

\section{Correspondência para:}

Fernanda Guglielmi Faustini Sonego e-mail: fgfsonego@unesc.net Universidade do Extremo Sul Catarinense Faculdade de Odontologia Av. Universitária, 1105, Bairro Universitário 88806-000 Criciúma/SC 